\title{
Establishment of a GFP Fluorescence Labeled GLP-1 Receptor Cell Line
}

\author{
Ming-Rui Li ${ }^{1}$, Xiu-Ling Ji ${ }^{1}$, Min Zhang ${ }^{1}$, Kwen-Jen Chang ${ }^{1}$, Dong-Bo Liu ${ }^{2}$, Yun-Lin Wei ${ }^{1}$ \\ ${ }^{1}$ Faculty of Life Science and Technology, Kunming University of Science and Technology, Kunming, Yunnan, 650005, \\ China \\ ${ }^{2}$ State Key Laboratory of Subhealth Intervention Technology, Hunan Agricultural University, Changsha, Hunan, China
}

\begin{abstract}
To establish a green fluorescent protein (GFP)based cellular model for screening GLP-1 receptor ligands from Traditional Chinese Medicinal herbs. A eukaryotic expression vector pCMV6-GLP-1R-GFP was constructed and transfected into U20S cells. A stable expressing GLP1R-GFP U20S cell line was established and the expression of GLP-1 receptor was confirmed by Western Blots with a GLP-1 specific antibody. The activities of expressed GLP-1GFP were investigated with the cellular redistribution of green fluorescence after stimulation with GLP-1 analogs in the absence or presence of a GLP-1 antagonist. The sensitivity and specificity of this cell line was qualified and suitable for GLP-1 receptor ligand screening.
\end{abstract}

Keywords-GLP-1receptor; GLP-1-GFP/U2OS cell line; Type2 diabetics.

\section{INTRODUCTION}

The glucagon-like peptide- 1 receptor (GLP-1R) is a therapeutically important family B G-protein-coupled receptor (GPCR) that is pleiotropically coupled to multiple signaling effectors, and with activities including regulation of insulin biosynthesis and secretion, GLP-1R is one of the key targets in the management of type II diabetes mellitus (DM) [1]. GLP-1 is an incretin hormone secreted by intestine L-cells in response to nutrient ingestion and is responsible for up to $70 \%$ of the insulin response after a meal [2]. After release, GLP-1 potentiates glucose-dependent insulin secretion [3], inhibits glucagon secretion [4], and increases $\beta$-cell mass by means of inducing $\beta$-cell proliferation and inhibiting the apoptosis of $\beta$-cell [5]. The release of GLP-1 further induces satiety, delays gastric emptying, and has potential effects in both cardiovascular and nervous systems. Compared with conventional treatment for type II DM, GLP-1 promotes insulin release only in the context of hyperglycemia, so that it does not cause hypoglycemia in human [6]. However, the promise of this receptor as a drug target for type II DM is hindered by the rapid degradation of endogenous peptides by dipeptidyl peptidase IV (DPPIV) in vivo. This has in part been overcome by the development of DPPIV-resistant GLP-1 mimetics such as exendin-4 and liraglutide as well as DPPIV inhibitors that prolong the plasma half-life of endogenous GLP-1R peptides $[7,8]$. Although these have therapeutic potential (indeed, exendin-4 is currently used clinically), frequent intravenous or subcutaneous administration are required for these GLP-1 analogs for clinical uses, which can reduce patients' compliance. In addition, exendin- 4 has also been associated with significant adverse side effects in some patients, including pancreatitis [9], of which the mechanistic basis is unknown. These difficulties have therefore driven the search for the development of small molecules orally active drugs that induce GLP-1R signaling.

In this study, GFP-tagged GLP-1R stably expressed cell lines was constructed, and the cell model was validated, we will use this GLP-1-GFP/U2 cells to screen small molecule ligands of GLP-1R from Yunnan abundant natural medicinal resources.

\section{MATERIALS AND METHODS}

\section{A. Materials}

Dulbecco's modified Eagle's medium (DMEM) and Fetal bovine serum (FBS) was purchased from Thermo Fisher Scientific (Melbourne, VIC, Australia). Cell culture flasks and plates were purchased from Costar. Lipofectamine 2000 transfection reagents were purchased from Invitrogen Corporation, G418 was purchased from Amresco Company; Exendin 4 drugs purchased from Eli Lilly, Exendin 9-39 purchased from Aladdin; human GLP$1 \mathrm{R}$ antibody was purchased from Abcam; eukaryotic expression vector pCMV6-AC-GFP was purchased from Origene; restriction endonuclease enzyme (SgfI, MluI) were purchased from Framentance; other reagents were domestic or imported analytical reagent.

\section{B. Methods}

1) Primer design

Primers were designed using primer premier 5.0 software.

Upstream primer: 5'-gaggcgatcgec ATGGCCGGCGCCCCCGGC-3 '; Downstream primer: 5'-gcgacgegtGCTGCAGGAGGCCTGGCAAG-3'.

In the upstream primer and downstream primer 5 'end plus a SgfI, MluI restriction sites and three protected bases (gag, gcg), and before the start codon plus the C, primers were synthesized by Shuo Yang technology company.

2) Human GLP-1R cDNA amplification

Human GLP-1R cDNA as a template, PCR amplification of full-length human GLP-1R cDNA coding region, length of $1392 \mathrm{bp}$. PCR reaction system : template $1 \mu \mathrm{l}, 10 \times$ Ex Taq Buffer $5 \mu \mathrm{l}$, dNTP $(2.5 \mathrm{mmol} / \mathrm{L})$ $3 \mu \mathrm{l}$, upstream and downstream primers $(10 \mathrm{pmol} / \mathrm{L})$ of each $1 \mu$, Ex Taq enzyme $1 \mu 1$, deionized water made up to $50 \mu \mathrm{l}$; reaction conditions : $95{ }^{\circ} \mathrm{C}$ denaturation $5 \mathrm{~min} ; 95{ }^{\circ} \mathrm{C}$ denaturation $45 \mathrm{~s}, 65{ }^{\circ} \mathrm{C}$ annealing $45 \mathrm{~s}, 72{ }^{\circ} \mathrm{C}$ extension $1 \mathrm{~min}$, a total of 30 cycles; $72{ }^{\circ} \mathrm{C}$ continue to extend $10 \mathrm{~min}$. The identification of the PCR product was confirmed by the agarose gel electrophoresis. 
3) The construction of human GLP-1R eukaryotic expression vector

PCR product and the pCMV6-AC-GFP plasmids were digested with restriction endonucleases SgfI and MluI respectively, $10 \mathrm{~g} / \mathrm{L}$ agarose gel electrophoresis, purified by gel extraction. After overnight incubation by the T4 DNA ligase and then transformed into E.coli DH5 $\alpha$, ampicillin culture, positive clones were picked after amplification for identification.

4) Establishment of stable transfection of human GLP1R-GFP/U2OS cell lines

U2OS cells maintained in DMEM medium with $10 \%$ fetal bovine serum and 1:100 (v/v) penicillin/streptomycin in a $5 \% \quad \mathrm{CO} 2$ saturated humidity training at $37^{\circ} \mathrm{C}$. pCMV6-AC-GFP and recombinant expression vector pCMV6-GLP-1R-GFP transfected by Lipofectamine2000 instructions. After transfection $48 \mathrm{~h}$, cells were digested with trypsin, selection medium with G418 $(400 \mu \mathrm{g} / \mathrm{ml})$ containing 2 weeks, the obtained positive clones with resistance to antibiotics, stably expressing the human GLP- 1R, named GLP-1R-GFP/U2OS cells.

5) Western-blot to detect the expression levels of GLP-1R-GFP translation

Transfected cells were collected and lysed, $40 \mu \mathrm{g}$ protein samples were treated by SDS-PAGE electrophoresis and electrically transferred to a PVDF membrane respectively, $50 \mathrm{~g} / \mathrm{L}$ skim milk shacked $2 \mathrm{~h}$ at room temperature, and 1: 200 diluted antibody overnight incubation at $4{ }^{\circ} \mathrm{C}$, PBST washing the membrane three times, each time $10 \mathrm{~min}$, then with 1: 5000 diluted secondary HRP-labeled antibody incubation $2 \mathrm{~h}$ at room temperature, PBST washing the membrane three times, each time $10 \mathrm{~min}, \mathrm{DAB}$ chromogenic detection of protein.

6) GLP-1 receptor activity analysis

GLP-1R-GFP/U2OS cells (1000 cells/well) were cultured in 96-well plates, cultured with $10 \%$ fetal bovine serum DMEM medium, $37^{\circ} \mathrm{C}, 5 \% \mathrm{CO} 2$ for 24 hours. The next day the medium is completely removed, washing with PBS buffer and added to the test sample Exendin-4 incubated for 20 minutes at room temperature, The redistribution of green fluorescence in GLP-1-GFP/U2OS cells in the presence of various concentrations of Exendin4 was observed using an inverted fluorescence microscope. The high content analysis system was used to analyze fluorescent spots number and average intensity.

\section{RESULTS}

\section{A. Identification of pCMV6-GLP-1R-GFP}

The recombinant plasmids (Fig. 1) were digested by SgfI and MluI, it can be found around $1.4 \mathrm{~kb}$ gene fragment size, using specific primers for the PCR amplification, the same gene fragment could be amplified, that confirming the foreign gene has been inserted into the vector. To further verify the insert size in the right direction or not, and recombinant plasmids were sequenced in both directions through the sequence alignment, it find the correct size and orientation.

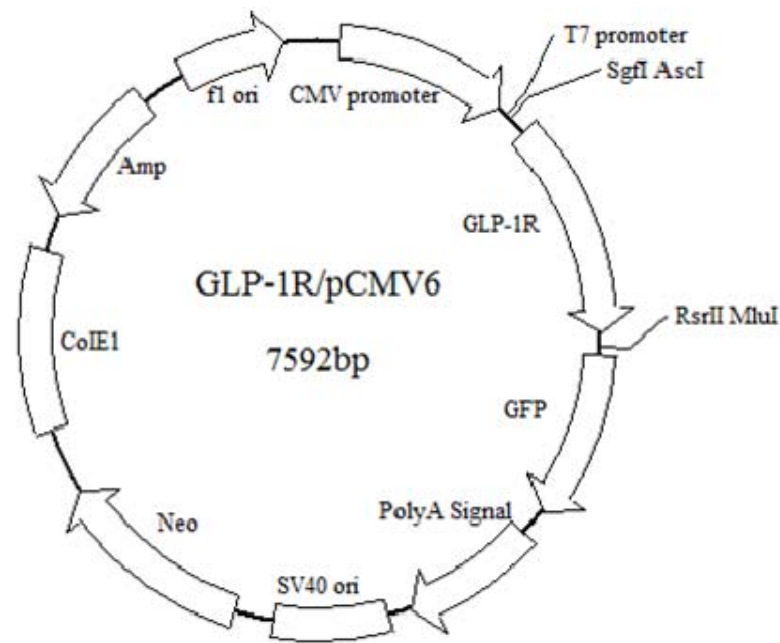

Figure 1. The recombinant plasmid pCMV6-GLP-1R-GFP

\section{B. GLP-1R-GFP/U2OS cell lines}

After transfection $24 \mathrm{~h}$, cells were trypsinized and cloned in a selection medium containing G418 $(400 \mu \mathrm{g} / \mathrm{ml})$ for 2 weeks, to obtain positive clones of resistance to antibiotic G418and expressed high level of GFP (Fig. 2). Expanding culture positive clones, that named GLP-1RGFP/U2OS cell lines. Western-blot test results showed that GLP-1R-GFP high level expression in cell lines GLP1R-GFP/U2OS cell lines.

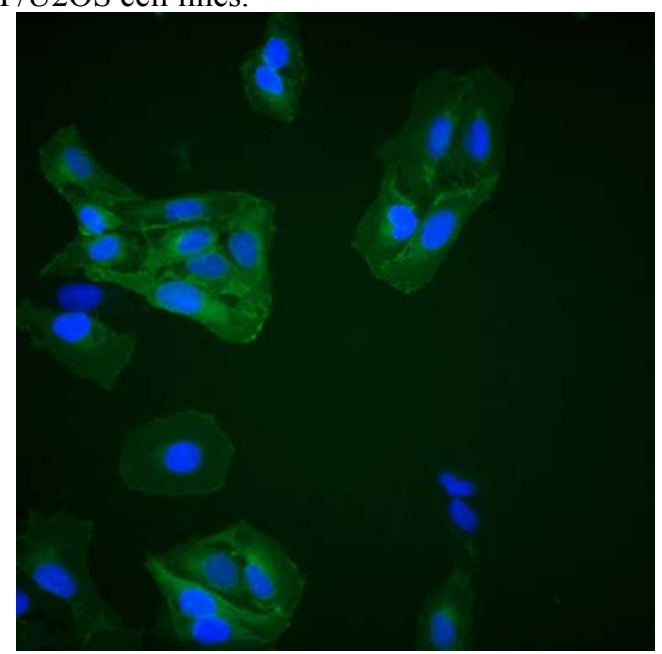

Figure 2. The obtained monoclonal cell line of GLP-1R-GFP/U2OS cells after G418 selection

\section{Verify GLP-1R-GFP/U2OS cell model}

The obtained GLP-1R-GFP/U2OS were cultured in 96-well culture plates (1000 cells/well) with $10 \%$ fetal bovine serum DMEM medium, $37{ }^{\circ} \mathrm{C}, 5 \% \mathrm{CO} 2$ for 24 hours. The next day the medium is completely removed, washing with PBS buffer and added to the test sample Exendin-4 and GLP-1 receptor antagonist (Exendin 9-39), incubated for 20 minutes, then fixed cells with $4 \%$ 
paraformaldehyde for 30 minutes at room temperature, washed with PBS buffer three times, finally nuclei were stained by adding the DAPI stain for 15 minutes, washed 5 times with PBS buffer, left $100 \mu$ L PBS buffer in each well. The cells were visualized under inverted fluorescence microscope to observe GFP green

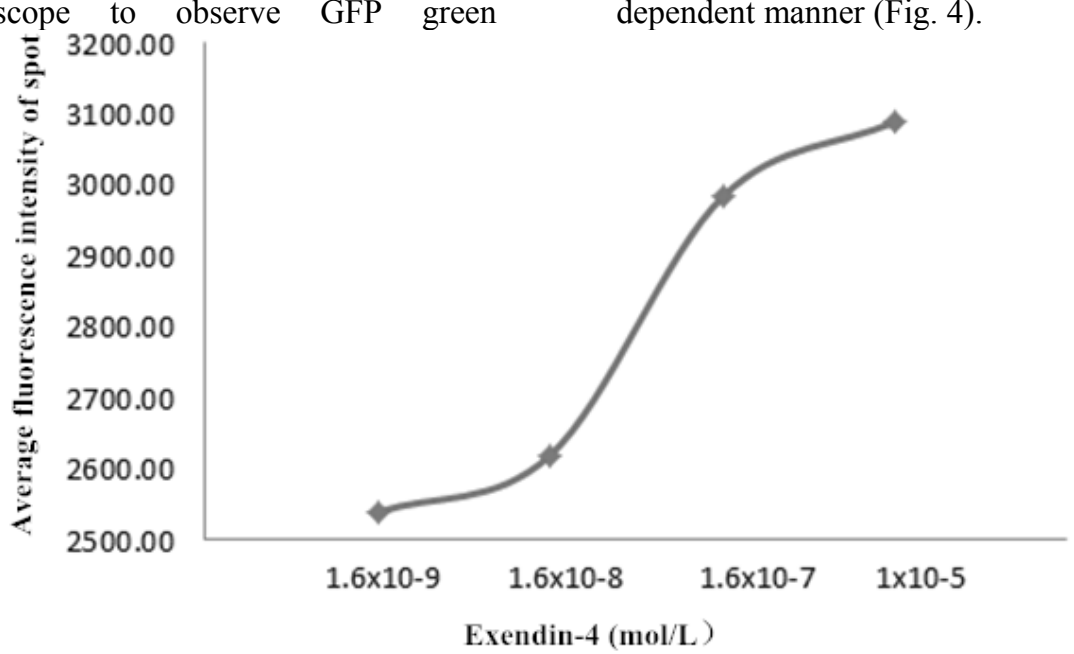

Figure 3. Concentration dependence of the increase in fluorescence intensity of bright spots after Exendin-4 treatment for 30 minutes. The average

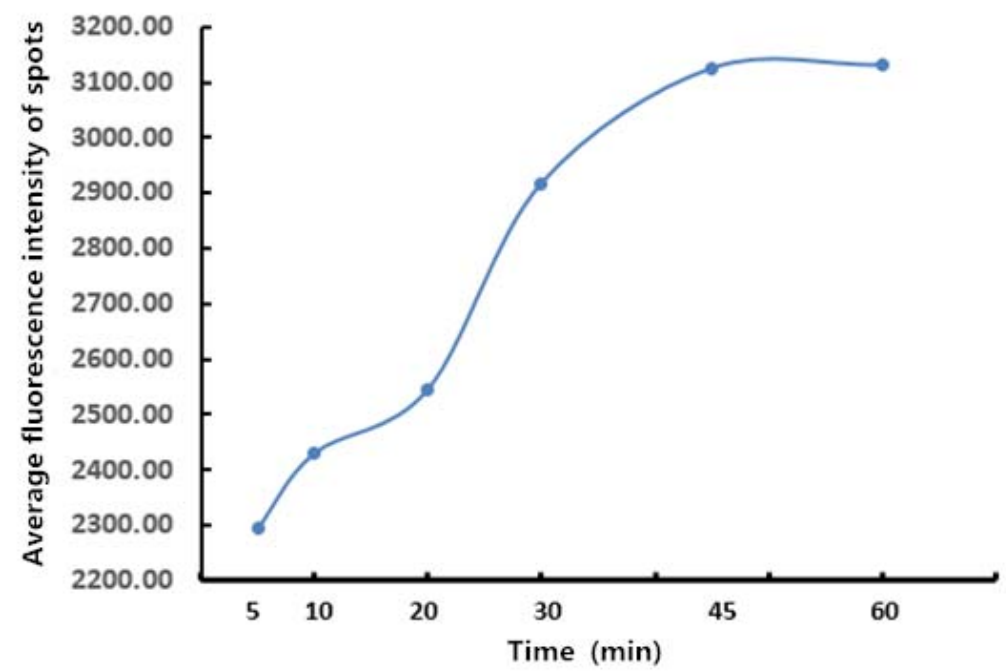

Figure 4 The time course of fluorescence image changes after Exendin- 4 treatment.

Top figure are the fluorescence image changes after various time of the addition of Exendin-4. Many tiny spots appeared after 15 minutes. At 60 minutes, many large bright spots (red color) appeared around nuclei (large green color) indicating that they are internalized vesicles. The bottom is the average fluorescence intensity of tiny spots acquired from high content analysis system. fluorescence spots with high content analysis system. The high content system analysis revealed the increase of fluorescent spots number and intensity in GLP-1RGFP/U2OS cells after the treatment with exendin-4 in a concentration-dependent manner (Fig. 3) and timedependent manner (Fig. 4).

\footnotetext{
fluorescence intensity of spot acquired from high content system.
}

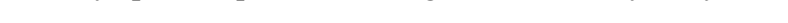

i


gene detection method is simple, qualitative and quantitative, and is suitable for high-throughput drug screening [13]. At present, the screening of GLP-1R receptor model mostly based on the downstream labeled cAMP response element coupled with CRE [14], by detecting the expression of the marker gene to effect reaction of the receptor with the ligand. Since many endogenous receptor activation will produce cAMP, so there is such a high false-positive, screening of the complex mixture of natural products cannot be achieved.

This study is the first to build a direct fluorescentlabeled human glucagon-like peptide-1 receptor (GLP-1R) cell screening model, compared with domestic and international screening method, it has rapid, sensitive, specific advantages, and will greatly reduce the occurrence of false positives, and can be used to screen for the complex mixture, but also reduce testing costs.

\section{REFERENCES}

[1] F.S. Williad and K.W. Sloop: Physiology and emerging biochemistry of the glucagon-like peptide-1 receptor. Exp Diabetes Res, 2012, 2012:Article ID 470851

[2] T. Vilsboll and J.J. Holst: Incretins, insulin secretion and type 2 diabetes mellitus incretins and diabetes. Diabetologia. 2004, 47, 357-366.

[3] B. Kreymann, G. Williams, M.A. Ghatei and S.R. Bloom: Glucagon-like peptide-1 7-36: a physiological incretin in man. Lancet. 1987, 2, 1300-1304.

[4] B.E. Dunning, J.E. Foley and B. Ahren: Alpha cell function in health and disease: influence of glucagon-like peptide-1. Diabetologia. 2005, 48, 1700-1713.
[5] R. Perfetti and H. Hui: The Role of GLP-1 in the life and death of pancreatic beta cells. Horm Metab. Res. 2004, 36, 804-810.

[6] M.A. Nauck, M.M. Heimesaat, K. Behle, J.J. Holst, M.S. Nauck, R. Ritzel, M. Hufner and W.H. Schmiegel: Effects of glucagon-like peptide 1 on counter regulatory hormone responses, cognitive functions, and insulin secretion during hyperinsulinemic, stepped hypoglycemic clamp experiments in healthy volunteers. Clin. Endocrinol. Metab. 2002, 87, 1239-1246.

[7] P. Nelson, T. Poon, X. Guan, C. Schnabel, M. Wintle and M. Fineman: The incretin mimetic exenatide as a monotherapy in patients with type 2 diabetes. Diabetes Technol Ther, 2007, 9: 317-326.

[8] M. Choy and S. Lam: Sitagliptin: a novel drug for the treatment of type 2 diabetes. Cardiol Rev, 2007, 15: 264-271.

[9] L. Olansky: Do incretin-based therapies cause acute pancreatitis? J Diabetes Sci Technol , 2010, 4:228-229.

[10] X.X. Zhao, R. Gao and F.R. Li: High through-put drug screening based on the cell model. Pharm Biotechnol, 2008, 15: 227-230.

[11] F. Guo, Y.X. Liu, D.X. Zheng and C.Y. Jiang: Establishment of high through-put drug screening model targeting retinoic acid receptor in cells. Acta Pharm Sin, 2005, 40: 908-911.

[12] M. Huang, Y. Rong, H.X. Ning, C.H. Wang, Y.Y. Wang and Z.J. Chang: Establishment of high through-put drug screening cell models based on JAK-STAT signal pathway. Acta Pharm Sin, 2004, 39: 164-167.

[13] O.G. Chepurny and G.G. Holz: A novel cyclic adenosine monophosphate responsive luciferase reporter incorporating a nonpalindromic cyclic adenosine monophosphate response element provides optimal performance for use in G protein coupled receptor drug discovery efforts. J Biomol Screen, 2007, 12: 740-746.

[14] G. Skoglund, M.A. Hussain and G.G. Hulz: Glucagon-like peptide 1 stimulates insulin gene promoter activity by protein kinase Aindependent activation of the rat insulin I gene cAMP response element. Diabetes, 2000, 49: 1156-1164. 